\title{
The circadian clock, metabolism and obesity
}

\author{
U. Albrecht
}

Department of Biology, Biochemistry, University of Fribourg, Fribourg, Switzerland

Address for correspondence: U. Albrecht, University of Fribourg, Department of Biology, Biochemistry, Chemin du Musée 5, 1700

Fribourg, Switzerland.

E-mail: urs.albrecht@unifr.ch

\begin{abstract}
Summary
In the last decades, obesity has been on the rise becoming a burden for health care systems. The reasons behind this rise are most likely caused by lifestyle rather than by an increase in gene mutations, because manifestations of genetic alterations would take longer than just a few decades. Lifestyle has a great impact on the circadian system and therefore on the body internal organization of physiological and biochemical processes, regulating various aspects of behavior and metabolism. In the following, I will discuss recent studies delineating relationships between metabolic processes and the circadian system, how metabolites and nutrients regulate the circadian clock and how nuclear receptors can act as metabolic sensors and clock regulators. Finally, I will discuss how clock modulation and feeding patterns influence the development of obesity.
\end{abstract}

Keywords: Fasting, feeding pattern, nuclear receptors, Sleep.

\section{Introduction}

On our planet, food is usually not available in a constant steady manner. There are periods of excess followed by periods of shortage. Therefore, evolution has shaped most organisms in a way that they can accumulate body fat as energy depots quite quickly in periods of nutritional excess in order to withstand periods of famine. This change in the availability of food is at least partially related to season and therefore to the length of the light period. Hence, the organism is adapting to changes in food availability and light period. Interestingly, these two natural parameters are not only changing over the year but also every day, defining the sleep and wake periods in mammals. During their activity phase mammals eat, whereas during the sleep phase they fast. In order to keep the blood glucose levels in a narrow almost constant range to provide the brain with its preferred fuel, metabolic adaptations are necessary. Because these changes are predictable and parallel the light dark cycle, a mechanism to predict both light and feeding changes developed in the form of a circadian clock mechanism that has a period of about one day. This circadian clock appears to have beneficial effects in a competitive environment as shown for cyanobacteria (1), plants (2) and mice (3). This clock mechanism is present in most cells of an organism and consists of a transcriptional-translational feedback loop made up by a set of clock genes, which activate (Bmal1, Clock) and inhibit (Per, Cry, Rev-erba) each other, thereby establishing a feedback loop with a period of about $24 \mathrm{~h} \mathrm{(4)} \mathrm{(Fig.} \mathrm{1A,} \mathrm{B).} \mathrm{In} \mathrm{order} \mathrm{to} \mathrm{produce} \mathrm{a} \mathrm{coherent} \mathrm{circa-}$ dian clock system, all the individual cellular clocks have to be synchronized in a tissue to make up tissue clocks. The various tissue and organ clocks in turn need to maintain a constant phase relationship to each other to build a stable systemic circadian oscillatory system that allows optimal preparation to daily recurring and predicable events that challenge the organism (5).

Disturbance of the phase relationships between organ clocks can result from performing shift work, which is accompanied by sleep restriction with concurrent circadian disruption. Epidemiological studies link short sleep duration and circadian disruption with higher risk for metabolic syndrome and diabetes (6). A recent study in humans showed that indeed exposure to prolonged sleep restriction with concurrent circadian disruption decreased the resting metabolic rate and increased plasma glucose concentration after a meal due to inadequate pancreatic insulin secretion (7). In mice, prolonged circadian disruption 


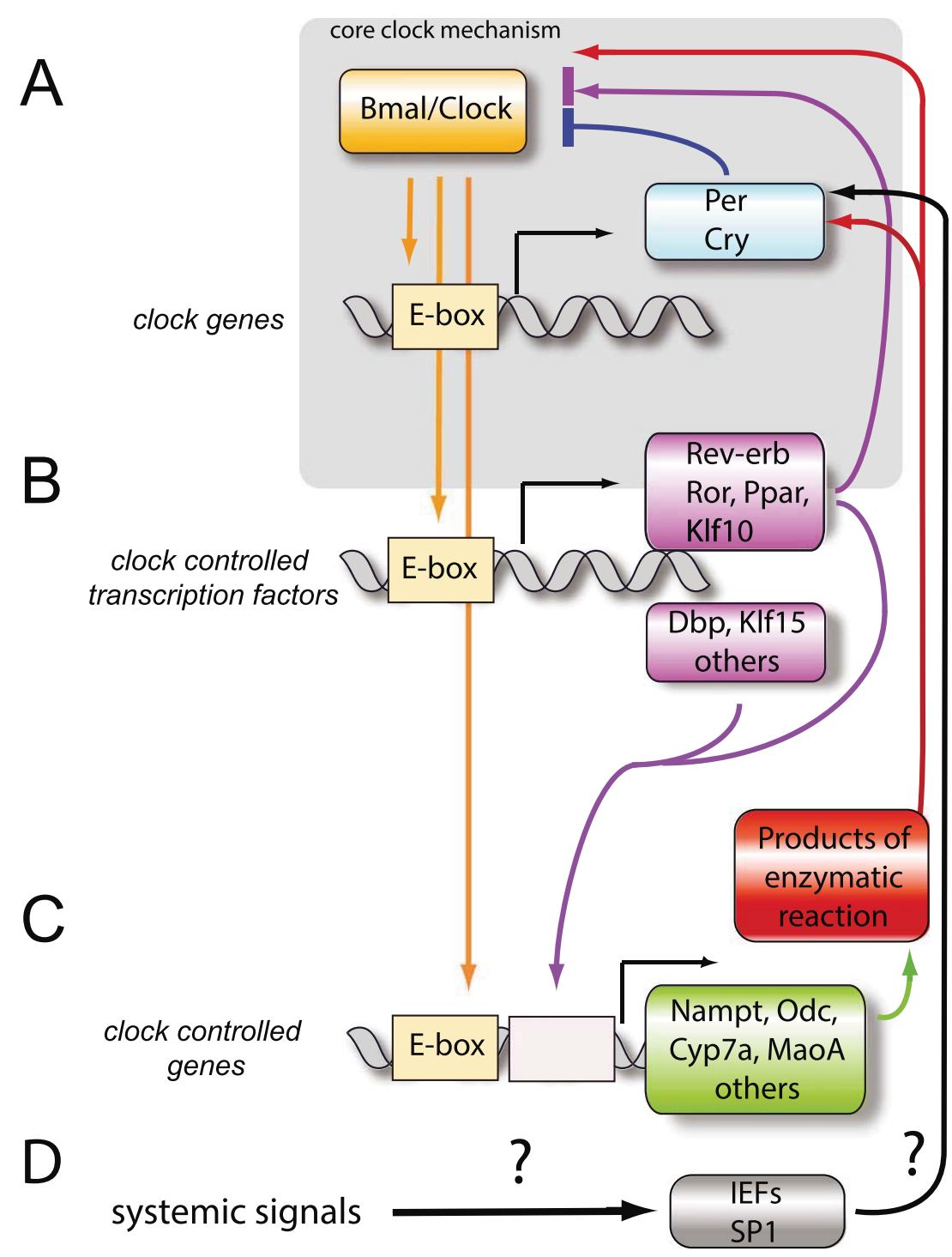

Figure 1 Molecular hierarchy and feedback mechanisms of the circadian clock in a cell. A) Core clock mechanism with the Bmal/Clock heterodimerbinding to E-box elements in the promoters of Per and Cry genes activating their transcription. Per and Cry proteins (blue) inhibit Bmal/Clock activity thereby turning off their own transcription. B) Bmal/Clock binds also to E-box elements present in the promoters of clock controlled transcription factors (purple). Rev-erb, Ror, Ppar and Klf10 either feed back and influence Bmal and Clock transcription or they can activate clock controlled genes via binding to their cognate promoters elements present for example in genes coding for rate-limiting enzymes. C) Clock controlled genes (ccgs) are either directly regulated by Bmal/Clock or indirectly via clock controlled transcription factors. These ccgs can code for enzymes whose products (red) can influence Bmal or Clock transcription. D) Systemic signals affect expression and activity of immediate early genes (IEFs) and the general transcription factor SP1, which may influence expression of Per genes and thereby clock phase and speed.

leads to adverse changes in the brain, manifested by dendritic reorganization and loss, altered responses to novel environments and increase in insulin levels and weight gain (8). Therefore, it is likely that changes in human behavior due to the industrial revolution lead to circadian de-synchrony and play an important if not dominant role in the pandemic of metabolic syndrome observed today (Fig. 2).

In the following sections, I will summarize the most recent observations that illustrate the relationships between the circadian clock and metabolic processes affecting important physiological functions of homeostasis.

\section{Relationships between metabolic processes and the circadian clock}

The basic nutritional energy carriers are carbohydrates, lipids and amino acids that are handled by cells to produce energy equivalents in the form of ATP. However, if cells do not require more ATP, the biochemical processes are 


\section{Synchrony}

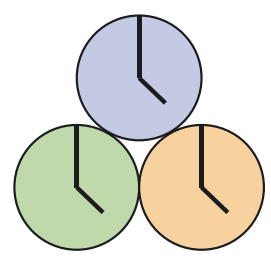

Disruption of a cycle

shift work

jet lag

stress

\section{Health}

Sleep/Wake Cycles Metabolic Cycles
Desynchrony

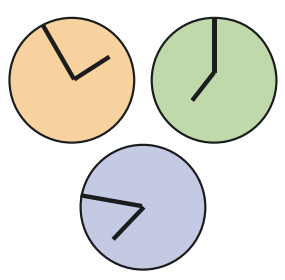

Disease

Figure 2 Desynchronization of the circadian system leads to disease. The circadian system is made up of multiple circadian cycles such as the sleep/wake cycle (blue), metabolic cycles (green) and feeding/fasting cycles (orange). In a healthy organism, they maintain a stable phase relationship to each other and hence are synchronized to each other. Disruption of synchrony can happen by disrupting one or more of the cycles, for example by rotating shift work, jet lag and stress. This leads to uncoupling of the cycles and subsequent development of disease such as metabolic syndrome, obesity and depression.

reversed, and energy is mainly stored in the form of glycogen and triglycerides. In order to avoid interference between catabolic and anabolic pathways, e.g. use of fatty acids (FAs) in $\beta$-oxidation and synthesis of FAs, cells can separate them spatially, e.g. in mitochondria versus the cytosol. Additionally, anabolic and catabolic pathways are separated temporally, because the organism's activity is structured in a time-of-day-dependent manner and hence metabolic needs are dramatically different during the activity and resting phase. High-throughput transcriptional studies revealed that approximately a tenth of the mammalian genes across various cell and tissue types exhibit $24-\mathrm{h}$ variations in mRNA levels. These transcripts code for proteins controlling processes such as mitochondrial oxidative phosphorylation, carbohydrate metabolism and transport, lipid biogenesis, and cholesterol synthesis and degradation (9). Additionally, about $20 \%$ of the proteome is subject to clock control with some post-translational modifications also cycling. Interestingly, a significant fraction of those proteins lack oscillations at the mRNA level (10), illustrating that cellular circadian oscillations are regulated at several levels of gene expression. Metabolite profiling adds additional complexity to the picture of circadian clock-regulated metabolic function, where $15 \%-70 \%$ of the metabolome in humans exhibits circadian variation (11).

At the molecular level, many genes coding for metabolically important enzymes are regulated directly or indirectly by components of the circadian clock mechanism (Fig. 1C). Direct regulation occurs via binding of BMAL1 and CLOCK to E-box elements in the promoters of a gene (Fig. 1), as for example in the gene coding for nicotinamide

phosphoribosyltransferase, the rate-limiting enzyme for adenine dinucleotide $\left(\mathrm{NAD}^{+}\right)$synthesis in the mammalian salvage pathway of nicotinamide $(12,13)$. Interestingly, $\mathrm{NAD}^{+}$ levels regulate the clock mechanism via SIRT1, a histone deacetylase that modulates transcriptional activity of BMAL1/CLOCK, creating a metabolic feedback-loop between the clock and metabolism $(12,14)$ (Fig. 1).

Indirect regulation of metabolic genes occurs via transcription factors that are regulated by the clock mechanism. These transcription factors include members of the PAR bZip family such as D site of albumin promoter binding protein (DBP), thyrotroph embryonic factor (TEF) and hepatic leukemia factor (HLF) (15) and nuclear receptors (16) that bind to their respective promoter elements, thereby activating genes regulating metabolism and hence serve as output regulators of the circadian clock. Interestingly, some of these transcription factors control the expression of clock components as for example DBP that regulates the Per1 gene (17) or REV-ERB $\alpha, \operatorname{ROR} \alpha$ and PPAR $\alpha$ that regulate Bmal1 (18-21). Hence, these transcription factors not only serve as clock output mediators but also as feedback control factors to the clock itself (Fig. 1). One of the puzzling observations in murine hepatocytes is, however, that despite the absence of BMAL1 or CLOCK, PER2 can continue to oscillate in a similar manner as in wild-type hepatocytes (22-24). This is in contrast to the current understanding of the clock mechanism based on experiments obtained studying the central clock of the suprachiasmatic nuclei (SCN) (Fig. 1A), indicating that peripheral clocks may differ at the mechanistic level from the central clock. Per 2 may be regulated by diurnally activated immediate early transcription factors (IEFs), such as serum response factor, glucocorticoid receptor (GR) and heat shock factor1 (HSF1) (25) as well as by SP1 that may compete with CLOCK and BMAL1. Because the amplitude of Per 2 expression is not reduced in BMAL1 or CLOCK-depleted mice, IEFs and SP1 are probably more efficient in stimulating Per2 transcription in peripheral tissues (26) (Fig. 1D). Of note is that GR is not expressed in the SCN and, hence, cannot interfere there with BMAL1/CLOCK mediated Per2 transcription (27).

Overall, these examples illustrate that the circadian clock and metabolic events are linked in self-balancing transcriptional networks; however, central and peripheral clock regulation may not be identical.

\section{Metabolites and nutrients regulating the circadian clock}

An important feature of the circadian clock is its adaptability to changes in the environment. Stimuli such as light and food can serve as signals to change clock phase in a tissue and eventually of the entire circadian system. For example, clock phase in the SCN is directly affected by light (reviewed in (28)) from where all the rest of the body clocks are then 
synchronized allowing the organism to adapt to the new light/dark cycle after jet lag. Food affects the phase of the liver clock permitting adaptation to changes in food availability. Interestingly, this food entrainment by the liver does not affect clock phase in the SCN (29), illustrating that periodic feeding signals, when not in phase with the light/dark cycle, can cause de-synchrony of the circadian system (Fig. 2).

Nutrient components have been identified as important factors to entrain peripheral circadian clocks (reviewed in (30), among which glucose appears to be a particularly potent entraining factor) (31). Fibroblasts treated with glucose displayed down-regulation of Per 1 and Per2 and as a consequence reduced clock resetting (32). Glucose up-regulates the negatively acting zinc-finger transcription factor KLF10 (TIEG1), which represses Bmal1 transcription (33). Interestingly, KLF10 is regulated by BMAL1/CLOCK and thus appears to be part of a feedback loop involving the circadian clock and glucose levels (34). A recent study indicates that glucose availability regulates cellular clock oscillation through the hexosamine/O-GlcNAc pathway by glycosylating BMAL1 and CLOCK leading to an increased stability of these proteins by inhibiting their ubiquitination (35). Furthermore, a combined injection of glucose and amino acids induced acute expression of multiple clock genes in the mouse liver (36), indicating that glucose may influence clock phase. For glucose homeostasis pancreatic beta cell function is essential. A recent study shows that pancreatic beta cells contain a circadian clock that controls insulin secretion (37), which is probably important to prepare the organism for predictable daily food intake and increase in blood glucose. Therefore, it appears that an organism's response to glucose is orchestrated by the circadian clock by providing a specific time window during which insulin secretion is optimal and hence more efficient, reducing the harmful effects of glucose. This may explain the impaired glucose tolerance in clock mutant mice (38), in which pancreatic cells do not work optimally (39).

Polyamines such as putrescine, spermidine and spermine modulate the interaction between PER2 and CRY1 proteins, resulting in the shortening of period of the circadian clock (40). Polyamines in the serum and clock period have an inverse relationship in the ageing process, with polyamines declining with age and clock period lengthening with age. Hence, dietary supplementation of polyamines may reverse the lengthening of clock period during the ageing process. Interestingly, the clock components BMAL1 and CLOCK directly regulate the expression of the gene for ornithine decarboxylase (Odc), which codes for the first and rate-limiting step of polyamine biosynthesis (40), highlighting a feedback loop between polyamine levels and the clock (Fig. 1C). Additional components of nutrition may affect the circadian clock either in a direct manner as described above or indirectly via binding to nuclear receptors that are either part or closely linked to the circadian clock mechanism $(16,18,21,41)$.

\section{Nuclear receptors as metabolic sensors and clock regulators}

A number of organic molecules, such as FAs, bile acids, oxysterols and glucocorticoids, serve as ligands for nuclear receptors, which go into the nucleus and bind to specific promoter elements of genes thereby regulating their expression in response to ligand binding. Many of these nuclear receptors display a diurnal expression profile (16) and the REV-ERBs and RORs are even part of the core clock mechanism (Fig. 1A, B; $(18,19,42,43)$. Deletion of REV-ERB $\alpha$ $(44,45)$ or double deletion of REV-ERB $\alpha$ and REV-ERB $\beta$ (42) resulted in metabolic alterations similar to those observed in Clock mutant or Bmal1 knock-out mice $(38,46)$, illustrating the close relationship between the circadian clock mechanism and metabolic regulation. Interestingly, however, REV-ERB $\alpha$ modulates the clock and metabolism by different genomic mechanisms. While clock control requires REV-ERB $\alpha$ to bind to the genome at its cognate site competing with ROR, metabolic genes are regulated by REV-ERB $\alpha$ through recruitment of the HDAC3 corepressor to sites to which it is bound by cell type-specific transcription factors (47). This allows REV-ERB $\alpha$ to impose a tissue-specific epigenomic rhythm that regulates metabolism customized to the specific need of a tissue (Fig. 1B). This differential regulation of the clock and metabolism by REV-ERB $\alpha$ raises the possibility that synthetic ligands that specifically affect REV-ERB $\alpha$ and NCoR/HDAC3 interaction without disrupting DNA binding could specifically alter liver metabolism with reduced side-effects on the circadian clock mechanism.

Glucocorticoids, produced by the cortex of the adrenal gland, regulating metabolic, cardiovascular and immunological functions, display a circadian rhythm in the circulation with peak levels during the onset of activity in humans (48) and rodents (49). Mice with mutations in clock components lose rhythmicity of their corticosterone levels $(50,51)$ indicating that the clock regulates glucocorticoids. In contrast, glucocorticoids can reset the phase of the circadian clock (27), via binding to the GR, which then translocates to the nucleus where it attaches to glucocorticoid response elements present in the promoters of several clock genes, including Per 1 and Per 2 . This suggests that GR is a critical regulator of clock gene expression (52). Interestingly, glucocorticoids do not act on the SCN itself, because it does not express GR; however, resetting of peripheral clocks by glucocorticoids depends on cues controlled by the SCN rather than on feeding-fasting rhythms (53). It is of note that CRY protein binds to GR and regulates its activity (41). This correlates with the observation that Cry1/ 2-double knock-out mice display an elevated response to 
glucocorticoids (41). Furthermore, these mice show increased levels of blood glucose, which parallels the deregulated activity of GR and increased expression of its metabolic target genes such as phosphoenolpyruvatecarboxykinase (Pepck). Interestingly, the CLOCK protein has also been shown to acetylate GR influencing the binding of GR to DNA (54).

Polyunsaturated FAs have been identified as ligands of peroxisome proliferator-activated receptors (PPARs) (reviewed in (55). Ligand activated PPARs regulate metabolic activities leading to FA catabolism, lipid storage and/or other effects, such as those affecting inflammation (56). In the liver, PPAR $\alpha$ is a master energy sensor (reviewed in (57). Its activity is impaired in mice lacking the three circadian PAR bZip factors DBP, HLF and TEF (58). Therefore, PPAR $\alpha$ is one of the links connecting the circadian clock and energy metabolism. In support of this view is the observation that the clock component PER2 interacts with PPAR $\alpha$ and serves as a co-regulator of this and other nuclear receptors (21). This interaction leads to rhythmic modulation of promoters of nuclear receptor target genes in vivo by PER2, like Bmal1, Hnf1 $\alpha$ and Glucose-6-phosphatase (21). For example, PPAR $\alpha$ binds to a peroxisome proliferator response element located in the promoters of uncoupling protein $1(25)$ and carnitin palmitoyl transferase 1 (59) on which its transcriptional activity is modulated by PER2 shaping the expression of these genes into a circadian profile. Interestingly, PPAR $\alpha$ also regulates Bmal1 expression establishing a feedback loop between the circadian clock and FA-mediated signaling (20) (Fig. 1A, B).

Taken together, glucocorticoid signaling via GR and FA signaling via PPAR $\alpha$ illustrate the intimate relationship between the clock, nuclear receptor signaling and metabolism.

\section{Clock modulation, feeding patterns and obesity}

As described above, the circadian clock is synchronized to the environment by light and feeding cues and is a body internal representation of the regular 24-h day. Light is sensed by the retina, from where intrinsically photosensitive retinal ganglion cells project via the retinohypothalamic tract directly to neurons of the SCN, evoking a signal that leads to changes in Per 1 and Per 2 clock gene expression. As a consequence, the phase of the circadian oscillator in the SCN is shifted eventually leading to a shift of the circadian system in the body (reviewed in (60)). Interestingly, endogenous glucocorticoid levels can be influenced by light. Light indirectly induces gene expression in the adrenal gland via the SCN-sympathetic nervous system, independent of glucocorticoid action on the hypothalmic-pituitary-adrenal axis (61), thereby submitting GR signaling at least partially to environmental lighting conditions. Traveling through several time zones is associated with corresponding changes in the lighting schedule and hence will shift the internal circadian rhythm of an organism accordingly. This shifting of the body internal clock phase to the new environmental phase takes time, and if this process is repeated regularly it leads to chronic de-synchrony of body functions which is associated with obesity. Mice exposed twice weekly to an advance of the LD cycle developed obesity when food was available ad libitum (62). Interestingly, however, timerestricted feeding to the initial dark phase prevented development of obesity (62).

Timing of the restricted access to food is critical and influences body weight. Restricted feeding of mice to daytime (resting phase) leads to weight gain (63) and misaligns the peripheral clocks with the SCN clock leading to metabolic alterations, which, on their own shift the peripheral circadian clock $(64,65)$. Conversely, restricting food access to the night (activity) phase can promote natural feeding rhythms and restore synchrony with circadian oscillations and prevent obesity $(66,67)$. Several studies showed that time-restricted feeding to the activity phase restores cycling of metabolic regulators such as CREB, mTOR and AMPK as well as oscillations of circadian clock genes and their targets. These parameters are all changed in diet-induced obesity but can be normalized by time-restricted feeding during the activity phase. Interestingly, mice on a high-fat diet become obese under ad libitum conditions, but under restricted feeding during the activity phase obesity is prevented, although the amount of calories consumed is similar to the ad libitum fed animals. Mice under the timerestricted feeding regimen displayed several abnormalities related to lipid metabolism (68-71). These positive effects of time-restricted feeding on metabolism could also be observed in other obesogenic diets such as high-fructose and high-sucrose diets (71). Furthermore, time-restricted feeding normalized metabolism in mice with pre-existing obesity and insulin resistance, and this was proportional to the length of time of food restriction; mice with restricted access to food for $9 \mathrm{~h}$ did better than those with access for $15 \mathrm{~h}(71)$.

Timed eating in humans suggests that weight-loss is affected by synchronizing feeding/fasting cycles with light/dark circadian rhythms (72). Individuals that underwent weight-loss treatment were more likely to lose weight when they consumed their calories earlier in the day compared to those who ate later (73). Similarly, a high-calorie breakfast is associated with better fasting glucose, insulin sensitivity and improved lipid profile compared to the effects of a high-calorie dinner (74). A study using a smartphone app to investigate eating patterns in the population revealed erratic eating patterns rather than the selfreported three daily meals. For half of the cohort, daily food intake duration exceeded $14.74 \mathrm{~h}$. Overweight, healthy individuals from this study were then following an eating schedule in which they could eat normally but only for 10-11 h daily. After 16 weeks, the participants reduced 
body weight and improved sleep, which persisted for a year (75). Taken together, time-restricted feeding has beneficial effects on the liver and other metabolic organs (Fig. 3).

A recent study suggests that circadian clock deficiency or chronic jet lag induce leptin resistance in mice, independent of obesity risk factors. The circadian clock appears to maintain long-term energy balance by controlling the leptin endocrine feedback loop between the adipose tissue and the brain, suggesting that circadian and metabolic synchrony may at least in part depend on leptin signaling (76). The circadian clock mechanism in white adipocytes may control leptin expression through BMAL1/CLOCK-modulated, $\mathrm{C} / \mathrm{EBP} \alpha$-mediated leptin gene transcription, although this modulation appears to be quite weak (76). Leptin resistance can also be induced by ER stress (77), and ER stress is activated by genetically induced circadian clock deficiency (78). Hence, leptin resistance may also be explained via ER stress in mice with circadian dysfunction. Genetic ablations of leptin or its receptor in mice support the notion that leptin signaling may affect the circadian clock. While leptindeficient mice display normal circadian period and altered photic resetting $(79,80)$ with disturbed peripheral clocks (81), leptin receptor-deficient animals show arrhythmicity in general activity (82) and slight alterations in Per2 mRNA levels in the SCN, and impairments in the hepatic molecular clockwork (83).

\section{PER2-a link between the circadian clock and systemic metabolic cues}

To establish circadian clock and metabolic synchrony, a sensor of both metabolic and clock state is most likely to play a key role. In an approach to discriminate genes whose cyclic expression is driven by the local liver clock or systemic signals, mice with a conditionally active liver clock were compared to mice in which the liver clock was inactive. Interestingly, the clock component Per2 did not depend on functional liver oscillators, and its expression continued to oscillate in their absence, indicating that Per2 oscillation in the liver is driven by systemic cues (23). Hence, Per2 may serve as both a sensor for cyclic systemic signaling cues and as a clock component. This makes Per 2 a good candidate to serve as a node and link between the circadian clock and systemic cues.

Subjecting animals to a timed feeding schedule with restricted food access for $4 \mathrm{~h}$ elicits food anticipatory activity (FAA) that is independent of the central clock in the SCN (84). Most of the genetically ablated circadian clock components in mice do not or only partially affect FAA (85-88) with the exception of Per2 (89). Hence, Per2 appears to be important for FAA. Interestingly, liver-specific, but not neuron-specific deletion of Per 2 leads to loss of FA by interfering with $\beta$-hydroxybutyrate $(\beta-\mathrm{OHB})$ production and its subsequent processing in the brain. However, timed $\beta$ $\mathrm{OHB}$ administration can only rescue FAA in liver-specific knock-out but not whole body knock-out mice, indicating the presence of a systemic feedback signaling depending on Per2 function also in other organs (59). Hence, Per2 may be a systemic integrator of metabolic cues as well as an effector to respond to these cues, which is in line with the view described above that IEFs and SP1 transcription factors may convey systemic information via Per2 activation to the clock, as for example shown for HSF1, which induces Per2 in response to cold exposure (25). Because timerestricted feeding is effective in the treatment of metabolic de-synchrony, one may speculate whether timed $\beta$-OHB administration may be helpful for night-eating disorder and related disorders in humans. Such experiments with mice
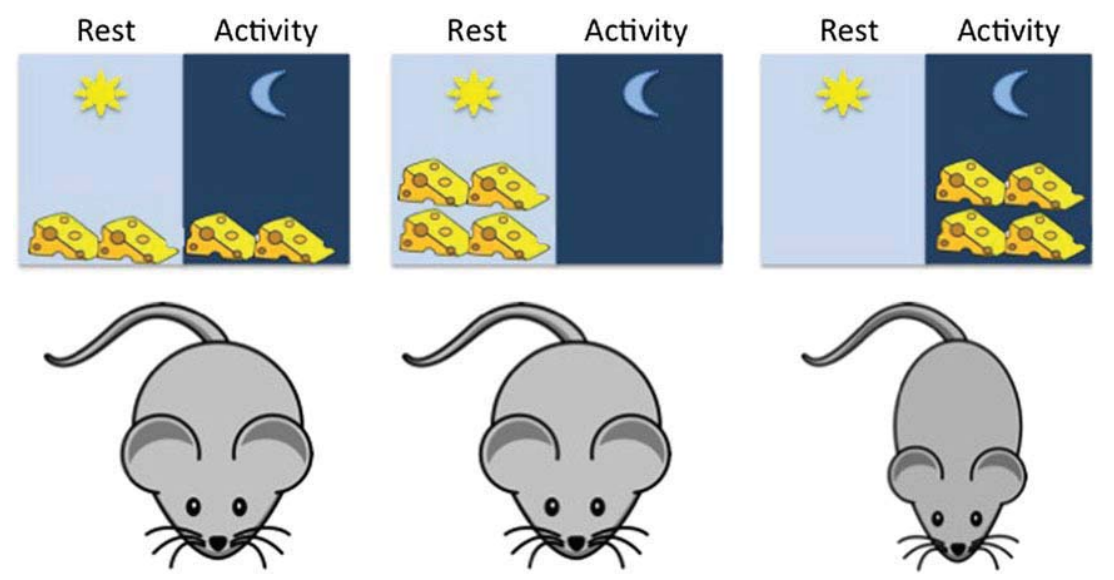

Figure 3 Effects of the feeding schedule on weight in mice. Left panel: Mice have ad libitum access to a high-fat diet. This leads to changes in the gut microbiome, stool metabolomics and hepatic gene expression. These mice are predisposed to obesity and dysmetabolism. Right panel: Time restricted feeding to the activity phase restores fluctuations in the gut microflora, luminal metabolites and hepatic gene expression. Probably, also oscillations in gut signaling are restored. These mice are protected against obesity and metabolic syndrome. Middle panel: Time restricted feeding to the resting phase misaligns the peripheral clocks with the master SCN clock and leads to metabolic syndrome. 
are underway and may reveal new avenues for treatment of derailed metabolic homeostasis.

\section{Conclusions}

Lifestyle has a great impact on body weight, and it appears that not only the amount of food but also the timing when food is taken up plays an important role in body weight regulation. The reason for this is the dynamic network organization of metabolism, which is controlled by three oscillating systemic networks; light-entrainable central oscillations in the brain, sleep/wake homeostasis and feeding/fasting rhythms (Fig. 2). Optimal efficiency of metabolic regulation is achieved when the systemic network oscillations are in a stable phase relationship with each other and therefore are synchronized. De-synchronization due to alternation of one of the networks lowers efficiency of metabolic regulation and therefore leads to the development of metabolic disease with obesity and diabetes as the most prominent manifestations. However, it is not clear whether de-synchronization is a hallmark in all forms of obesity. Future studies will also have to address how circadian rhythms and feeding/fasting cycles co-operate and what the mechanisms of interaction are. This is also related to the question how de-synchrony leads to metabolic disease and what the molecular signaling pathways are that lead to metabolic derailment. One of the obvious unresolved questions is, how homeostatic satiety regulation, involving the brain hypothalamic nuclei and peripheral organs such as the gut, is affected by the circadian clock and how its disruption and/or de-synchronization from metabolic cycles affects homeostatic satiety regulation. The gut microbiome displays cyclic changes according to the feeding/fasting rhythms, and it appears to contribute to the development of obesity and metabolic disease. However, it is not understood how the gut microbiome affects rhythms in metabolism and physiology.

\section{Conflict of interest statement}

I declare no conflict of interest.

\section{Acknowledgements}

I thank Drs. Jürgen Ripperger and Abdul Dulloo for comments on the manuscript. This work was supported by the Swiss National Science Foundation and the State of Fribourg.

\section{References}

1. Ouyang Y, Andersson CR, Kondo T, Golden SS, Johnson CH. Resonating circadian clocks enhance fitness in cyanobacteria. Proc Natl Acad Sci U S A 1998; 95: 8660-8664.
2. Dodd AN, Salathia N, Hall A et al. Plant circadian clocks increase photosynthesis, growth, survival, and competitive advantage. Science 2005; 309: 630-633.

3. Spoelstra K, Wikelski M, Daan S, Loudon AS, Hau M. Natural selection against a circadian clock gene mutation in mice. Proc Natl Acad Sci U S A 2016; 113: 686-691.

4. Partch CL, Green CB, Takahashi JS. Molecular architecture of the mammalian circadian clock. Trends Cell Biol 2014; 24: 90-99. 5. Mohawk JA, Green CB, Takahashi JS. Central and peripheral circadian clocks in mammals. Annu Rev Neurosci 2012; 35: 445-462.

6. Punjabi NM, Shahar E, Redline S et al. Sleep-disordered breathing, glucose intolerance, and insulin resistance: the Sleep Heart Health Study. Am I Epidemiol 2004; 160: 521-530.

7. Buxton OM, Cain SW, O'Connor SP et al. Adverse metabolic consequences in humans of prolonged sleep restriction combined with circadian disruption. Sci Transl Med 2012; 4: 129 ra143.

8. Karatsoreos IN, Bhagat S, Bloss EB, Morrison JH, McEwen BS. Disruption of circadian clocks has ramifications for metabolism, brain, and behavior. Proc Natl Acad Sci U S A 2011; 108: 1657-1662.

9. Panda S, Antoch MP, Miller BH et al. Coordinated transcription of key pathways in the mouse by the circadian clock. Cell 2002; 109: 307-320.

10. Koike N, Yoo SH, Huang HC et al. Transcriptional architecture and chromatin landscape of the core circadian clock in mammals. Science 2012; 338: 349-354.

11. Dallmann R, Viola AU, Tarokh L, Cajochen C, Brown SA. The human circadian metabolome. Proc Natl Acad Sci U S A 2012; 109: 2625-2629.

12. Nakahata Y, Sahar S, Astarita G, Kaluzova M, Sassone-Corsi P. Circadian control of the NAD+ salvage pathway by CLOCKSIRT1. Science 2009; 324: 654-657.

13. Ramsey KM, Yoshino J, Brace CS et al. Circadian clock feedback cycle through NAMPT-mediated NAD+ biosynthesis. Science 2009; 324: 651-654.

14. Asher G, Gatfield D, Stratmann M et al. SIRT1 regulates circadian clock gene expression through PER2 deacetylation. Cell 2008; 134: 317-328.

15. Gachon F, Olela FF, Schaad O, Descombes P, Schibler U. The circadian PAR-domain basic leucine zipper transcription factors DBP, TEF, and HLF modulate basal and inducible xenobiotic detoxification. Cell Metab 2006; 4: 25-36.

16. Yang X, Downes M, Yu RT et al. Nuclear receptor expression links the circadian clock to metabolism. Cell 2006; 126: 801-810.

17. Mitsui S, Yamaguchi S, Matsuo T, Ishida Y, Okamura H. Antagonistic role of E4BP4 and PAR proteins in the circadian oscillatory mechanism. Genes Dev 2001; 15: 995-1006.

18. Preitner N, Damiola F, Lopez-Molina L et al. The orphan nuclear receptor REV-ERBalpha controls circadian transcription within the positive limb of the mammalian circadian oscillator. Cell 2002; 110: 251-260.

19. Sato TK, Panda S, Miraglia LJ et al. A functional genomics strategy reveals Rora as a component of the mammalian circadian clock. Neuron 2004; 43: 527-537.

20. Canaple L, Rambaud J, Dkhissi-Benyahya O et al. Reciprocal regulation of brain and muscle Arnt-like protein 1 and peroxisome proliferator-activated receptor alpha defines a novel positive feedback loop in the rodent liver circadian clock. Mol Endocrinol 2006; 20: 1715-1727.

21. Schmutz I, Ripperger JA, Baeriswyl-Aebischer S, Albrecht U. The mammalian clock component PERIOD2 coordinates circadian output by interaction with nuclear receptors. Genes Dev 2010; 24: 345-357. 
22. Debruyne JP, Noton E, Lambert CM, Maywood ES, Weaver DR, Reppert SM. A clock shock: mouse CLOCK is not required for circadian oscillator function. Neuron 2006; 50: 465-477.

23. Kornmann B, Schaad O, Bujard H, Takahashi JS, Schibler U. System-driven and oscillator-dependent circadian transcription in mice with a conditionally active liver clock. PLoS Biol 2007; 5: e34.

24. Kornmann B, Schaad O, Reinke H, Saini C, Schibler U. Regulation of circadian gene expression in liver by systemic signals and hepatocyte oscillators. Cold Spring Harb Symp Quant Biol 2007; 72: 319-330.

25. Chappuis S, Ripperger JA, Schnell A et al. Role of the circadian clock gene Per2 in adaptation to cold temperature. Mol Metab 2013; 2: 184-193.

26. Schibler U, Gotic I, Saini C et al. Clock-talk: interactions between central and peripheral circadian oscillators in mammals. Cold Spring Harb Symp Quant Biol 2015 .pii: 027490

27. Balsalobre A, Brown SA, Marcacci L et al. Resetting of circadian time in peripheral tissues by glucocorticoid signaling. Science 2000; 289: 2344-2347.

28. Ramkisoensing A, Meijer JH. Synchronization of biological clock neurons by light and peripheral feedback systems promotes circadian rhythms and health. Front Neurol 2015; 6: 128.

29. Damiola F, Le Minh N, Preitner N, Kornmann B, Fleury-Olela F, Schibler U. Restricted feeding uncouples circadian oscillators in peripheral tissues from the central pacemaker in the suprachiasmatic nucleus. Genes Dev 2000; 14: 2950-2961.

30. Froy $\mathrm{O}$. The relationship between nutrition and circadian rhythms in mammals. Front Neuroendocrinol 2007; 28: 61-71.

31. Stephan FK, Davidson AJ. Glucose, but not fat, phase shifts the feeding-entrained circadian clock. Physiol Behav 1998; 65: 277-288.

32. Hirota T, Okano T, Kokame K, Shirotani-Ikejima H, Miyata T, Fukada Y. Glucose down-regulates Per1 and Per2 mRNA levels and induces circadian gene expression in cultured Rat-1 fibroblasts. J Biol Chem 2002; 277: 44244-44251.

33. Hirota T, Kon N, Itagaki T, Hoshina N, Okano T, Fukada Y. Transcriptional repressor TIEG1 regulates Bmal1 gene through GC box and controls circadian clockwork. Genes Cells 2010; 15: $111-121$

34. Guillaumond F, Grechez-Cassiau A, Subramaniam M et al. Kruppel-like factor KLF10 is a link between the circadian clock and metabolism in liver. Mol Cell Biol 2010; 30: 3059-3070.

35. Li MD, Ruan HB, Hughes ME et al. O-GlcNAc signaling entrains the circadian clock by inhibiting BMAL1/CLOCK ubiquitination. Cell Metab 2013; 17: 303-310.

36. Oike H, Nagai K, Fukushima T, Ishida N, Kobori M. Feeding cues and injected nutrients induce acute expression of multiple clock genes in the mouse liver. PLoS One 2011; 6: e23709.

37. Perelis M, Marcheva B, Ramsey KM et al. Pancreatic beta cell enhancers regulate rhythmic transcription of genes controlling insulin secretion. Science 2015; 350: aac4250.

38. Turek FW, Joshu C, Kohsaka A et al. Obesity and metabolic syndrome in circadian Clock mutant mice. Science 2005; 308: 1043-1045.

39. Marcheva B, Ramsey KM, Buhr ED et al. Disruption of the clock components CLOCK and BMAL1 leads to hypoinsulinaemia and diabetes. Nature 2010; 466: 627-631.

40. Zwighaft Z, Aviram R, Shalev M et al. Circadian clock control by polyamine levels through a mechanism that declines with age. Cell Metab 2015; 22: 874-885.

41. Lamia KA, Papp SJ, Yu RT et al. Cryptochromes mediate rhythmic repression of the glucocorticoid receptor. Nature 2011; 480: 552-556.
42. Cho H, Zhao X, Hatori $\mathrm{M}$ et al. Regulation of circadian behaviour and metabolism by REV-ERB-alpha and REV-ERB-beta. Nature 2012; 485: 123-127.

43. Bugge A, Feng D, Everett LJ et al. Rev-erbalpha and Reverbbeta coordinately protect the circadian clock and normal metabolic function. Genes Dev 2012; 26: 657-667.

44. Delezie J, Dumont S, Dardente H et al. The nuclear receptor REV-ERBalpha is required for the daily balance of carbohydrate and lipid metabolism. FASEB J 2012; 26: 3321-3335.

45. Gerhart-Hines Z, Feng D, Emmett MJ et al. The nuclear receptor Rev-erbalpha controls circadian thermogenic plasticity. Nature 2013; 503: 410-413.

46. Rudic RD, McNamara P, Curtis AM et al. BMAL1 and CLOCK, two essential components of the circadian clock, are involved in glucose homeostasis. PLoS Biol 2004; 2: e377.

47. Zhang Y, Fang B, Emmett MJ et al. Gene regulation. Discrete functions of nuclear receptor Rev-erbalpha couple metabolism to the clock. Science 2015; 348: 1488-1492.

48. Weitzman ED, Fukushima D, Nogeire C, Roffwarg H, Gallagher TF, Hellman L. Twenty-four hour pattern of the episodic secretion of cortisol in normal subjects. J Clin Endocrinol Metab 1971; 33: 14-22.

49. Cheifetz PN. The daily rhythm of the secretion of corticotrophin and corticosterone in rats and mice. J Endocrinol 1971; 49: xi-xii.

50. Oster H, Damerow S, Kiessling S et al. The circadian rhythm of glucocorticoids is regulated by a gating mechanism residing in the adrenal cortical clock. Cell Metab 2006; 4: 163-173.

51. Dallmann R, Touma C, Palme R, Albrecht U, Steinlechner S. Impaired daily glucocorticoid rhythm in Per1 (Brd) mice. J Comp Physiol A Neuroethol Sens Neural Behav Physiol 2006; 192: 769-775.

52. So AY, Bernal TU, Pillsbury ML, Yamamoto KR, Feldman BJ. Glucocorticoid regulation of the circadian clock modulates glucose homeostasis. Proc Natl Acad Sci U S A 2009; 106: 17582-17587. 53. Le Minh N, Damiola F, Tronche F, Schutz G, Schibler U. Glucocorticoid hormones inhibit food-induced phase-shifting of peripheral circadian oscillators. EMBO J 2001; 20: 7128-7136.

54. Nader N, Chrousos GP, Kino T. Circadian rhythm transcription factor CLOCK regulates the transcriptional activity of the glucocorticoid receptor by acetylating its hinge region lysine cluster: potential physiological implications. FASEB J 2009; 23: 1572-1583.

55. Wahli W, Michalik L. PPARs at the crossroads of lipid signaling and inflammation. Trends Endocrinol Metab 2012; 23: 351-363.

56. Varga T, Czimmerer Z, Nagy L. PPARs are a unique set of fatty acid regulated transcription factors controlling both lipid metabolism and inflammation. Biochim Biophys Acta 1812; 2011: $1007-1022$.

57. Montagner A, Rando G, Degueurce G, Leuenberger N, Michalik L, Wahli W. New insights into the role of PPARs. Prostaglandins Leukot Essent Fatty Acids 2011; 85: 235-243.

58. Gachon F, Leuenberger N, Claudel T et al. Proline- and acidic amino acid-rich basic leucine zipper proteins modulate peroxisome proliferator-activated receptor alpha (PPARalpha) activity. Proc Natl Acad Sci U S A 2011; 108: 4794-4799.

59. Chavan R, Feillet C, Fonseca Costa SS et al. Liver-derived ketone bodies are necessary for food anticipation. Nature Commun 2016; 7: 10580.

60. Golombek DA, Rosenstein RE. Physiology of circadian entrainment. Physiol Rev 2010; 90: 1063-1102.

61. Ishida A, Mutoh T, Ueyama $\mathrm{T}$ et al. Light activates the adrenal gland: timing of gene expression and glucocorticoid release. Cell Metab 2005; 2: 297-307. 
62. Oike H, Sakurai M, Ippoushi K, Kobori M. Time-fixed feeding prevents obesity induced by chronic advances of light/dark cycles in mouse models of jet-lag/shift work. Biochem Biophys Res Commun 2015; 465: 556-561.

63. Arble DM, Bass J, Laposky AD, Vitaterna MH, Turek FW. Circadian timing of food intake contributes to weight gain. Obesity (Silver Spring) 2009; 17: 2100-2102.

64. Mukherji A, Kobiita A, Chambon P. Shifting the feeding of mice to the rest phase creates metabolic alterations, which, on their own, shift the peripheral circadian clocks by 12 hours. Proc Natl Acad Sci U S A 2015; 112: E6683-E6690.

65. Mukherji A, Kobiita A, Damara $\mathrm{M}$ et al. Shifting eating to the circadian rest phase misaligns the peripheral clocks with the master SCN clock and leads to a metabolic syndrome. Proc Natl Acad Sci U S A 2015; 112: E6691-E6698.

66. Salgado-Delgado R, Angeles-Castellanos M, Saderi N, Buijs RM, Escobar C. Food intake during the normal activity phase prevents obesity and circadian desynchrony in a rat model of night work. Endocrinology 2010; 151: 1019-1029.

67. Hatori M, Vollmers C, Zarrinpar A et al. Time-restricted feeding without reducing caloric intake prevents metabolic diseases in mice fed a high-fat diet. Cell Metab 2012; 15: 848-860.

68. Sherman H, Genzer Y, Cohen R, Chapnik N, Madar Z, Froy $\mathrm{O}$. Timed high-fat diet resets circadian metabolism and prevents obesity. FASEB J 2012; 26: 3493-3502.

69. Zarrinpar A, Chaix A, Yooseph S, Panda S. Diet and feeding pattern affect the diurnal dynamics of the gut microbiome. Cell Metab 2014; 20: 1006-1017.

70. Tsai JY, Villegas-Montoya C, Boland BB et al. Influence of dark phase restricted high fat feeding on myocardial adaptation in mice. J Mol Cell Cardiol 2013; 55: 147-155.

71. Chaix A, Zarrinpar A, Miu P, Panda S. Time-restricted feeding is a preventative and therapeutic intervention against diverse nutritional challenges. Cell Metab 2014; 20: 991-1005.

72. Mattson MP, Allison DB, Fontana L et al. Meal frequency and timing in health and disease. Proc Natl Acad Sci U S A 2014; 111: 16647-16653.

73. Garaulet M, Gomez-Abellan P, Alburquerque-Bejar JJ, Lee YC, Ordovas JM, Scheer FA. Timing of food intake predicts weight loss effectiveness. Int J Obes (Lond) 2013; 37: 604-611.

74. Jakubowicz D, Barnea M, Wainstein J, Froy O. High caloric intake at breakfast vs. dinner differentially influences weight loss of overweight and obese women. Obesity (Silver Spring) 2013; 21: 2504-2512.

75. Gill S, Panda S. A smartphone app reveals erratic diurnal eating patterns in humans that can be modulated for health benefits. Cell Metab 2015; 22: 789-798.
76. Kettner NM, Mayo SA, Hua J, Lee C, Moore DD, Fu L. Circadian dysfunction induces leptin resistance in mice. Cell Metab 2015; 22: 448-459.

77. Pan H, Guo J, Su Z. Advances in understanding the interrelations between leptin resistance and obesity. Physiol Behav 2014; 130: 157-169.

78. Cretenet G, Le Clech M, Gachon F. Circadian clockcoordinated $12 \mathrm{Hr}$ period rhythmic activation of the IRE1alpha pathway controls lipid metabolism in mouse liver. Cell Metab 2010; 11: 47-57.

79. Grosbellet E, Gourmelen S, Pevet P, Criscuolo F, Challet E. Leptin normalizes photic synchronization in male ob/ob mice, via indirect effects on the suprachiasmatic nucleus. Endocrinology 2015; 156: 1080-1090.

80. Sans-Fuentes MA, Diez-Noguera A, Cambras T. Light responses of the circadian system in leptin deficient mice. Physiol Behav 2010; 99: 487-494.

81. Ando H, Kumazaki M, Motosugi Y et al. Impairment of peripheral circadian clocks precedes metabolic abnormalities in ob/ob mice. Endocrinology 2011; 152: 1347-1354.

82. Grosbellet E, Dumont S, Schuster-Klein C et al. Circadian phenotyping of obese and diabetic $\mathrm{db} / \mathrm{db}$ mice. Biochimie 2015 .pii: S0300-9084(15)00208-4

83. Kudo T, Akiyama M, Kuriyama K, Sudo M, Moriya T, Shibata S. Night-time restricted feeding normalises clock genes and Pai-1 gene expression in the $\mathrm{db} / \mathrm{db}$ mouse liver. Diabetologia 2004; 47: $1425-1436$

84. Stephan FK, Swann JM, Sisk CL. Anticipation of 24-hr feeding schedules in rats with lesions of the suprachiasmatic nucleus. Behav Neural Biol 1979; 25: 346-363.

85. Pitts S, Perone E, Silver R. Food-entrained circadian rhythms are sustained in arrhythmic Clk/Clk mutant mice. Am J Physiol Regul Integr Comp Physiol 2003; 285: R57-R67.

86. Storch KF, Weitz CJ. Daily rhythms of food-anticipatory behavioral activity do not require the known circadian clock. Proc Natl Acad Sci U S A 2009; 106: 6808-6813.

87. Dudley CA, Erbel-Sieler C, Estill SJ et al. Altered patterns of sleep and behavioral adaptability in NPAS2-deficient mice. Science 2003; 301: 379-383.

88. Iijima M, Yamaguchi S, van der Horst GT, Bonnefont X, Okamura H, Shibata S. Altered food-anticipatory activity rhythm in Cryptochrome-deficient mice. Neurosci Res 2005; 52: 166-173.

89. Feillet CA, Ripperger JA, Magnone MC, Dulloo A, Albrecht U, Challet E. Lack of food anticipation in Per2 mutant mice. Curr Biol 2006; 16: 2016-2022. 